\title{
Deletion of myeloid-PTP1B decreases MHC Class I expression and peptide presentation through an IL-10 dependent mechanism in response to LPS challenge
}

\author{
Samantha Le Sommer ${ }^{*}$, Cristina Martin-Granados, Mirela Delibegovic
}

From 1st Annual Meeting of the Scottish Society of Cytomics (SCC) 2014. "Translational Cytometry from Bench to Bedside"

Aberdeen, UK. 25 September 2014

Protein Tyrosine Phosphatase 1B (PTP1B) inhibition is a target in the treatment of type 2 Diabetes Mellitus, and as such PTP1B inhibitors are in Phase II clinical trials. Previously our laboratory demonstrated that myeloid-specific deletion of PTP1B (LysM PTP1B) results in an increase in systemic IL-10 secretion and expression. In the current work we investigated how PTP1B deficiency affects the activation phenotype of murine macrophages in response to inflammatory stimuli. We demonstrate that myeloidspecific PTP1B deletion results in a decrease in expression of MHC Class I, along with co-stimulatory molecules CD80 and CD40. Interaction assays reveal a defect in the cells' ability to activate reporter B3Z T cells. Myeloidspecific PTP1B deletion increases the percentage of bone-marrow-derived-macrophages (BMDMs) positive for IL-10 which is associated with a decrease in iNOS production. Western blotting analysis demonstrated hyperphosporylation of ERK1/2 which has been suggested before to improve access to the IL-10 promoter. This provides evidence to suggest that myeloid-PTP1B deletion decreases MHC Class I expression and peptide presentation through an IL-10-dependent mechanism.
Published: 16 April 2015

doi:10.1186/1476-9255-12-S1-P1

Cite this article as: Le Sommer et al:: Deletion of myeloid-PTP1B decreases MHC Class I expression and peptide presentation through an IL-10 dependent mechanism in response to LPS challenge. Journal of Inflammation 2015 12(Suppl 1):P1.
* Correspondence: r02sI13@abdn.ac.uk

Institute of Medical Sciences (IMS) School of Medical Sciences, University of Aberdeen, UK 